Case Report

\title{
A rare case of invasive fungal sinusitis by co-infection of two fungi in a post COVID-19 patient: a case report
}

\author{
Timna Chungath*, Deepak Dileep Kumar, Shalu Susan Mathew, Jijo Paul
}

Department of ENT, Sanjivini multi-specialists Hospital, Alappuzha, Kerala, India

Received: 01 June 2021

Accepted: 16 July 2021

\section{*Correspondence:}

Dr. Timna Chungath,

E-mail:Idrtimna@yahoo.com

Copyright: ( $)$ the author(s), publisher and licensee Medip Academy. This is an open-access article distributed under the terms of the Creative Commons Attribution Non-Commercial License, which permits unrestricted non-commercial use, distribution, and reproduction in any medium, provided the original work is properly cited.

\begin{abstract}
Invasive fungal rhinosinusitis is a disease of the paranasal sinuses and nasal cavity that typically affects immunocompromised patients in acute fulminant form. Early symptoms can often mimic rhino sinusitis while late symptoms can cause significant morbidity and mortality. With early diagnosis and treatment can increase the patient survival. This case report is of 73 years old diabetic post COVID-19 patients. The patient presented to us was having severe headache and facial pain confined to left side cheek area with loosening of teeth. The patient underwent nasal cavity and biopsy from the maxillary sinus (left side) under local anaesthesia, which confirmed the diagnosis of invasive fungal sinusitis suggestive of co-infection by 2 fungi, aspergillus flavus and mucor. Further, the patient underwent surgical debridement and started on liposomal amphotericin B and patient improved significantly.
\end{abstract}

Keywords: COVID-19, Immuno compromised, Invasive fungal sinusitis, Necrosis, KOH, Debridement, Liposomal amphotericin B

\section{INTRODUCTION}

Invasive fungal sinusitis is a well described clinical entity characterised by the mucosal infiltration of mycotic organisms and angiocentric extension into orbital and intracranial structures. The population at risk for invasive fungal rhino sinusitis is with immunocompromised diseases and those on increased doses of immune suppressive therapies. ${ }^{1}$

Invasive fungal sinusitis can be classified into acute invasive and chronic invasive forms. The spores of these fungi enter through mouth and nose of the patient. Immunocompetent individuals mount a macrophage response, which will phagocytose these spores, and thus they don't develop the disease. Immunocompromised patients are unable to mount such a response allowing germination of spores into hyphae, which result in vascular invasion. ${ }^{2}$
Common causes of underlying immune suppression include diabetes mellitus, especially those with ketoacidosis, neutropenia patients, patients with haematological malignancies, history of organ transplants, bone marrow transplants patients, chemotherapy induced neutropenia and patients with advanced AIDS. Aspergillus infection is commonly seen in neutropenic patients and in diabetic patients commonly seen fungi are zygomycetes species like mucor, ascidia, Rhizopus. ${ }^{3}$

Early changes are mucosal thickening with quick progression into necrotic tissue in the sinuses spreading into nasal cavity, palate, orbit, cavernous sinus, cranial nerves, skull base, carotid artery and brain. Diagnosis of invasive fungal sinusitis often mandates imaging studies along with clinical endoscopic and histo-pathological confirmation. Treatment consists of antifungal therapy, aggressive surgical debridement with reversal of underlying immune suppressive condition. ${ }^{4}$ 


\section{CASE REPORT}

This case report is a 73 years old patient who had COVID-19 infection earlier and was treated as an inpatent and discharged home. The patient came after 2 weeks with severe headache and facial pain with cheek swelling all confined to left side of the face for 4 days duration. The patient is diabetic with history of myocardial infarction in the past.

Patient had taken MRI scan with contrast, which showed evidence of fungal sinusitis in the left maxillary sinus and erosion of posterolateral antral wall, floor of the orbit and infra orbital canal.

On nasal endoscopic examination, deviation of nasal septum towards left side. Left side middle meatus was filled with greyish black debri. Fungal debri were cleared from the middle meatus and maxillary antrostomy done under local anaesthesia. The maxillary sinus was filled with fungal debri was cleared and sent for $\mathrm{KOH}$ smear and fungal culture. Mucosal biopsy taken from maxillary sinus mucosa, which was necrotic for histopathological examination.

$\mathrm{KOH}$ smear showed evidence of aseptate broad hyphae suggestive of zygomycetes and fungal culture revealed growth of aspergillus flavus and mucor species. Histopathological revealed necrotising inflammatory lesion with fungal hyphae suggestive of mucormycosis.

Patient treatment was started with liposomal amphotericin B, endoscopic surgical debridement was done and patient improved significantly thereafter.

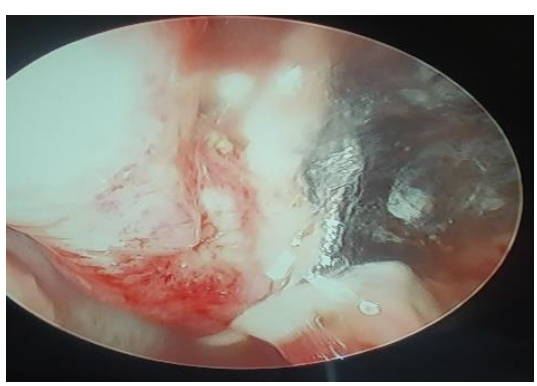

Figure 1: Maxillary sinus mucosa escharotic.

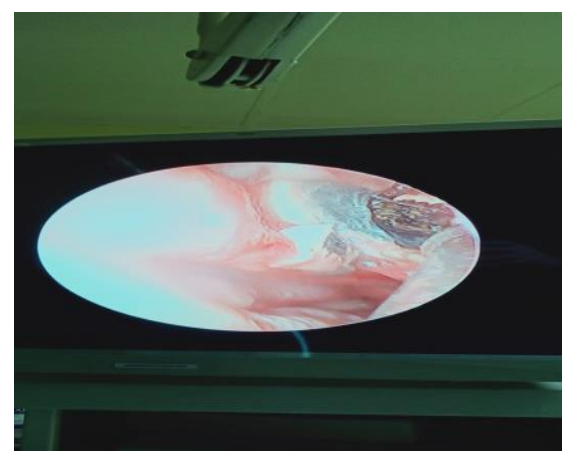

Figure 2: Fungal debri in maxillary sinus.

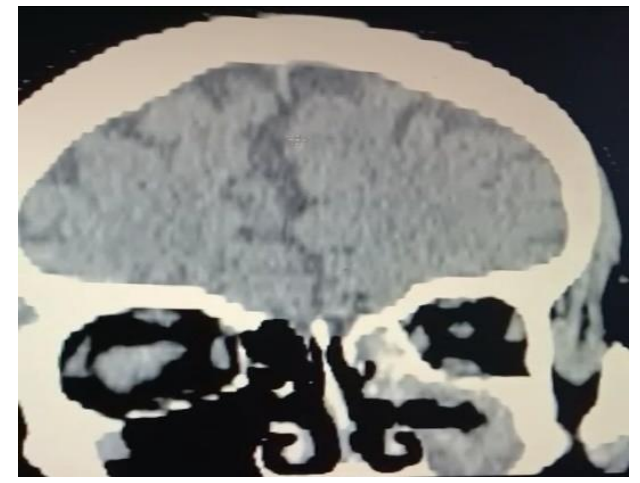

Figure 3: CT scan of paranasal sinuses.

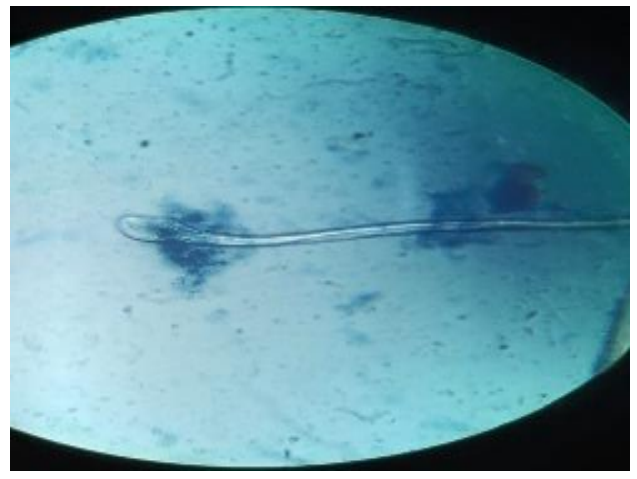

Figure 4: Fungal smear.

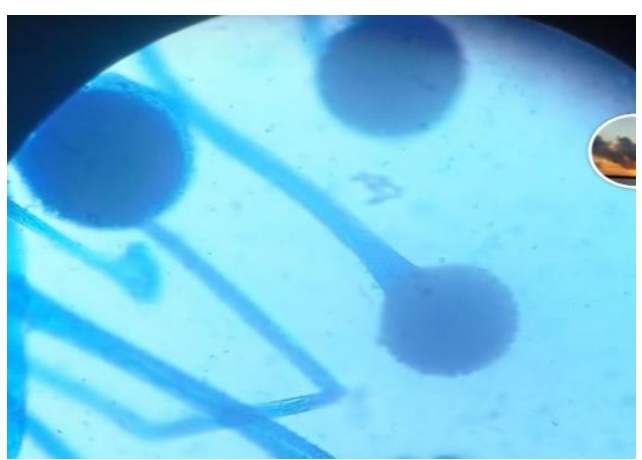

Figure 5: Fungal culture.

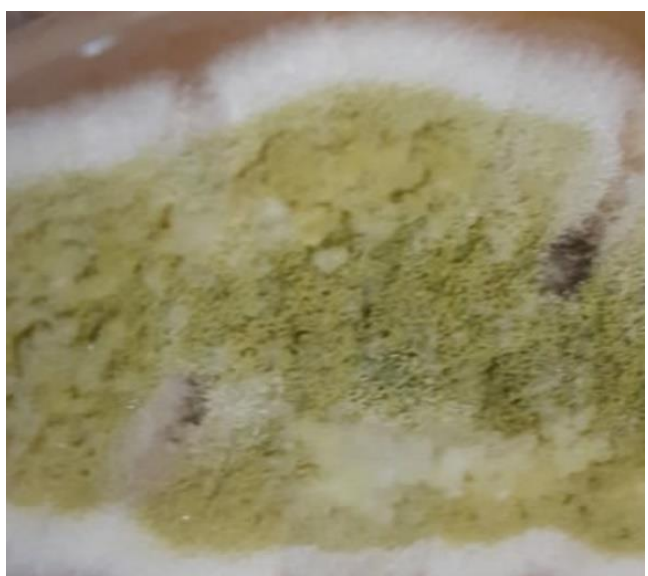

Figure 6: Fungal culture on gross of 2 fungi. 


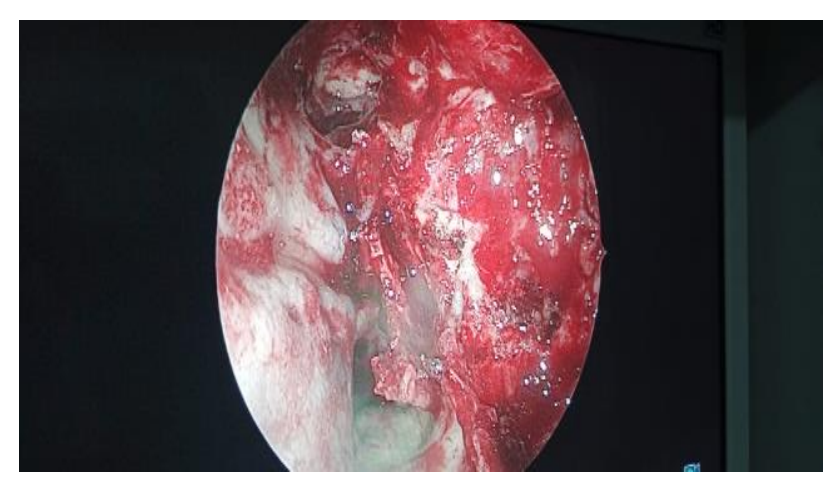

Figure 7: Maxillary sinus, post debridement.

\section{DISCUSSION}

Fungal rhinosinusitis can be broadly classified to 2 varieties, non-invasive and invasive. Non-invasive types are fungal ball and allergic, whereas in-vasive types are of with acute invasive and chronic invasive. Chronic invasive is of 2 types granulomatous and nongranulomatous types. These manifestations may progress from non-invasive to invasive form if the immunological status of the patient worsens. ${ }^{5}$

Acute fulminant type is usually caused by mucorales and aspergillus fumigates. Chronic invasive form is by aspergillus fumigatus and chronic granulomatous form by aspergillus flavus. The main presenting symptoms are headache, fever, epistaxis, nasal septal, palatal eschars, cough, and mental status changes. ${ }^{6}$

Diagnostic features in imaging studies are- in CT scan of paranasal sinuses, severe unilateral mucosal thickening and involvement of periantral fat planes. Involvement of pterygopalatine fossa has also been described. The Middle turbinate, maxillary sinuses, ethmoid air cells, sphenoid sinuses are commonly affected and the frontal sinus is less frequently affected. Bony dehiscence, orbital invasion, intra cranial extension are more specific features but uncommon in early disease. ${ }^{7}$

Most likely the initial site of the disease is to be the anterior aspect of middle turbinate and suggesting diagnosis during the initial biopsies in this location. ${ }^{8}$ The diagnosis is confirmed on histopathological evaluation if the fungal elements are found invading the mucosa, blood vessels, soft tissues or bone. ${ }^{9}$

$\mathrm{KOH}$ smear-one drop of $20 \% \mathrm{KOH}$ is applied over the specimen and covered with a cover-glass, $10 *$ to $40 *$ magnification is used to visualise the hyphae. ${ }^{10}$ Fungal culture-all mucorales grows rapidly in 3-5 days on Sabouraud agar or potato dextrose agar when incubated at $25-30^{\circ} \mathrm{c}$. mucorales are broad aseptate irregular ribbon like hyphae. ${ }^{11}$

Surgical treatment-includes surgical resection of all the necrotic tissues, systemic antifungal antibiotics and reversal of immune dysfunction. The goal of surgical treatment is to remove all the necrotic nonviable tissues. ${ }^{12}$ Intra venous antifungal antibiotics are the mainstay of treatment, with liposomal amphotericin B is the empirical drug of choice against zygomycetes and aspergillus isolates. $^{13}$

The newer azole Posaconazole is a choice, which covers zygomycetes and aspergillus species and it can be given orally. ${ }^{14}$ In those with neutropenia, granulocyte monocyte colony stimulating factor is a good choice to augment the immune system. ${ }^{15}$

\section{CONCLUSION}

In the case of COVID-19 pandemic, especially the second wave by the mutant virus strain in India resulted in post-COVID sequelae of invasive mycosis in already immunosuppressed individuals such as with diabetic, post chemo therapy, cancer patients, those undergoing dialysis and those with have history of organ transplants. Because of the significant suppression of immune system, the fungal spores germinate and grow into this dangerous condition. Zygomycetes and aspergillus are the commonest ones causing these sequelae. Diagnosis relies on histology and fungal culture of the tissues from involved sites helps in identification at species level. CT with contrast scan of the para nasal sinuses is more sensitive for bony changes. MRI with contrast taken to see the intra orbital and intra cranial extension of the disease. Aggressive surgical debridement along with systemic antifungal treatment is the treatment. Liposomal amphotericin B is the fungistatic drug for zygomycetes. It is the US food and drug administration, which approved the drug as a choice for invasive zygomycetosis. Serial nasal endoscopy is mandatory to check on the progress of the disease after debridement.

\section{Funding: No funding sources \\ Conflict of interest: None declared \\ Ethical approval: Not required}

\section{REFERENCES}

1. Boyd Gillespie M, Bert W, Malley O. An approach to fulminant invasive fungal rhino sinusitis in the immunocompromised host. Arch otolaryngol head and neck surg. 1998;124(5)520-6.

2. Invasive fungal sinusits: an overview. Otorhinolaryngol clin: an int j. 2009;1(1)45-7.

3. Feky M, Gaillard F. Acute invasive fungal sinusitis Radopedis.

4. Duggal P. In chapter 8 Invasive fungal rhino sinusitis. Am J Rhnol Allergy. 2013.

5. Gupta AK, Bansal S. Invasive fungal sinusits. Clin rhinol-an int J. 2012;5(2)63-71.

6. Deshazo RD. Syndromes of invasive fungal sinusitis. Med mycol. 2009;47(1):s309-14.

7. Middle Brooks EH, Frost CJ. Acute invasive fungal rhinosinsitis- a comprehensive update of CT findings 
and design of an effective diagnostic imaging model. Am j neuroradiol. 2015;36(8)1529-35.

8. Gillepsie MB, Malley BW. An approach to fulminant invasive fungal rhinosinusitis in the immunocompromised host. Arch otolaryngol head neck surg. 1998;124:520-26.

9. De Shazo RD, Chaplin K Swain RE. Fungal sinusitis. N Engl J Med. 1997;337:254-9.

10. Ponka D. Microscopic potassium hydroxide preparation can. Fam physician. 2014;60(1):57.

11. Lackner M, Caramelho R. Laboratory diagnosis of mucormycosis- current status and future perceptives future. Microbiol. 2014;9(5)683-95.

12. Epstein VA, Keen RC. Invasive fungal sinusitis and complications of rhinosinusitis, otolaryngol clnics North Am. 2008;41:497-524.

13. Moen MO, Lyseng, Williamson KA, Scott LJ. Liposomal amphotericin $\mathrm{B}$ a review of its use as empirical therapy in febrile neutropenia and in the treatment of fungal infection. Drugs. 2009;69:36192.

14. Langner S, Staber PB, Neimeister P. Posaconazole in the management of refractory invasive fungal infections. Thor clin risk management. 2008;4:74758.

15. Offner F. Haematopoietic growth factor in cancer patients with invasive fungal infections. Eur $\mathbf{J}$ clin microbiol infectious disease. 1997;16:56-63.

Cite this article as: Chungath T, Kumar DD,

Mathew SS, Paul J. A rare case of invasive fungal sinusitis by co-infection of two fungi in a post COVID-19 patient-a case report. Int J

Otorhinolaryngol Head Neck Surg 2021;7:1361-4. 